\title{
Olaparib in the therapy of advanced ovarian cancer: first real world experiences in safety and efficacy from China
}

\author{
Jing $\mathrm{Ni}^{1 \dagger}$, Xianzhong Cheng ${ }^{1 \dagger}$, Rui Zhou ${ }^{1}, \mathrm{Xia}_{\mathrm{X}} \mathrm{Xu}^{2}$, Wenwen Guo ${ }^{3}$ and Xiaoxiang Chen ${ }^{1 *}$ (D)
}

\begin{abstract}
Purpose: Poly (ADP-ribose) polymerase (PARP) inhibitor, is a milestone in treatment of ovarian cancer. However, there is no real world study from China regarding the clinical outcome of the taking PARP inhibitor (PARPi), Olaparib(Lynparza $\left.{ }^{\mathrm{TM}}\right)$. The goal of this research is to evaluate the side effects and short-term efficacy in advanced ovarian cancer patients who administered Olaparib.
\end{abstract}

Methods: Patients with ovarian cancer, fallopian tube cancer and peritoneal cancer that treated with Olaparib in The Affiliated Cancer Hospital of Nanjing Medical University between September 2018 and June 2019 were recruited. The drug associated Adverse Events (AEs) were collected and short-term efficacy were analyzed by modified Response Evaluation Criteria in Solid Tumors (mRECIST) .

Results: Of all 28 enrolled patients, 92.9\% were ovarian cancer, $7.1 \%$ were fallopian tube cancer, and 39.3\% cases harbored germline BRCA-mutation. There were 6(21.4\%) patients received Olaparib after multi-line chemotherapy, and 10 patients (35.7\%) as second-line maintenance therapy and 2 patients (7.1\%) as first-line maintenance therapy. There were still other 10 cases (35.7\%) received Olaparib as exploratory therapy. Abdominal distention, decreased blood pressure, increased body hair, thirsty, burning sensation of stomach and leg swelling were newly reported AEs. Serious Adverse Events(SAEs) were usually managed by dose interruption or dose reduction, rather than discontinuation. 3 patients discontinued treatment, 8 patients received reduced dose of Olaparib, and 4 patients stopped therapy after the alleviation of AEs. Of all 28 enrolled cases, in monotherapy group, 1 of 6 patients achieved stable disease(SD) and also 2 patients achieved stable disease(SD) combined with anti-angiogenic drugs when disease progressed. 2 patients achieved complete remission(CR) and 3 patients were stable with exploratory therapy.

Conclusions: The AEs of Olaparib were all manageable. For the first time, we also identified several AEs such as abdominal distention, decreased blood pressure, increased body hair, thirsty, burning sensation of stomach and leg swelling during the follow-up which have not been reported. The short-term efficacy was observed in some exploratory cases that provided new potential indication to PARPi-related clinical trials.

Keywords: Olaparib, Ovarian cancer, Safety;short-term efficacy

\footnotetext{
* Correspondence: cxxxxcyd@gmail.com

${ }^{\dagger}$ Jing $\mathrm{Ni}$ and Xianzhong Cheng contributed equally to this work.

'Department of Gynecologic Oncology, The Affiliated Cancer Hospital of

Nanjing Medical University, Jiangsu Cancer Hospital, Jiangsu Institute of

Cancer Research, 42\# Baiziting Street, Nanjing, Jiangsu 210009, People's

Republic of China

Full list of author information is available at the end of the article
}

(c) The Author(s). 2019 Open Access This article is distributed under the terms of the Creative Commons Attribution 4.0 International License (http://creativecommons.org/licenses/by/4.0/), which permits unrestricted use, distribution, and reproduction in any medium, provided you give appropriate credit to the original author(s) and the source, provide a link to the Creative Commons license, and indicate if changes were made. The Creative Commons Public Domain Dedication waiver (http://creativecommons.org/publicdomain/zero/1.0/) applies to the data made available in this article, unless otherwise stated. 


\section{Background}

Ovarian cancer accounts for about 4\% of cancer deaths among women worldwide, and is the most lethal gynecological malignancy [1]. In 2019, it is estimated that there will be approximately 22,530 cases of new identified ovarian cancer, and more than 13, 980 women will die from it in the United States [2]. The number of new cases of ovarian cancer in China reached 52,100 in 2015, of which about 22,500 died [3]. The vast majority (>90\%) of ovarian malignancies are epithelial ovarian cancer (EOC), and most patients are diagnosed as FIGO III/IV. The 5-year survival rate of ovarian cancer is about $30 \%$. Currently, the standard treatment for advanced epithelial ovarian cancer is maximal cytoreductive surgery and platinum-based chemotherapy [4].

Although the majority of patients with ovarian cancer can benefit from the first-line platinum-based chemotherapy, about $80 \%$ of patients will relapse within 1 to 2 years and suffer multiple recurrences, and patients gradually develop into platinum resistance ovarian cancer [5]. Therefore, it is a burning issue to extend progression-free period and thus improve the 5-year survival rate.

Poly adenosine diphosphate ribosome polymerase (PARP) is a DNA repair enzyme that plays a key role during DNA repair. PARP is activated when DNA is damaged and broken. As a receptor of DNA damage, PARP can recognize and bind to where DNA breaks, and then activate and catalyze the ribosylation of receptor proteins. PARPi inhibits the repair processes of DNA single-strand damage that can be transferred to double-strand damage (DSB) during the formation of DNA replication fork. Also, DSB can be repaired by homologous recombination (HR) pathway. When homologous recombination repair defects present in tumor cells (such as BRCA1 and BRCA2 mutations) that make DSB damage unrepairable, PARP inhibitors and homologous recombination repair defects react in the lethal synthesis of tumor cells [6].

Olaparib (Lynparza ${ }^{\mathrm{TM}}$ ) is the first-in-class oral PARPi. Previous studies have indicated that ovarian cancer patients with germline BRCA mutations platinum-resistant to multi-line chemotherapy could be benefit from Olaparib monotherapy with median progression-free survival (PFS) 7 months and overall survival(OS)16.6 months [7]. Both Study 19 and SOLO2 showed that Olaparib maintenance therapy significantly increased PFS without any detrimental effect on quality of life for those patients with no BRCA-mutated or BRCA-mutated platinum-sensitive recurrent serous ovarian cancer respectively [8, 9]. Further, the favorable results of SOLO1 showed that
Olaparib provided a substantial clinical benefit among women in newly diagnosed advanced ovarian cancer with a BRCA1/2 mutation [10]. Based on above mentioned clinical trials, new models and methods for the treatment of ovarian cancer are introduced.

Currently, Olaparib is approved for maintenance treatment of platinum-sensitive relapsed ovarian cancer in many regions, such as the United States, Europe and China [10]. Moreover, it can still be used for maintenance treatment of BRCA mutation after remission of first-line platinum chemotherapy in the United States, Europe and Japan, for monotherapy of germline mutation after third-line chemotherapy only in the United States [11]. Up to present, there is no relevant report of real world data on the administration of Olaparib from China. In this study, the adverse events and short-term effects of Olaparib for patients with ovarian cancer in the real world were retrospectively analyzed.

\section{Materials and methods Study population}

Present study was approved by the ethics committee of The Affiliated Cancer Hospital of Nanjing Medical University. Informed consent was obtained from all involved participants. Patients with ovarian cancer, fallopian tube cancer or primary peritoneal cancer that were treated with Olaparib (Lynparza ${ }^{\mathrm{TM}}$ ) between September 2018 to June 2019 at our cancer center were enrolled. All patients took Olaparib for more than 28 days and were followed up with CA125 or imaging examination. We recorded the basic characteristics of these patients, including the age, Eastern Cooperative Oncology Group performance status (ECOG PS) before the start of the treatment, histological type of the primary lesion, history, clinical stage on the basis of Federation International of Gynecology and Obstetrics (FIGO), BRCA mutation, history of therapy before and after the using of Olaparib and the follow-up. Safety was monitored by recording patients, chief complaint, physical examinations, vital signs, adverse events, as well as hematology and clinical chemistry tests.

\section{Group standard}

According to the National Comprehensive Cancer Network (NCCN) 2019.V1 guidelines for ovarian cancer, patients who progresses during initial treatment, or completely alleviates after initial treatment (cytoreductive surgery and platinum-based chemotherapy), but recur within 6 months are defined as platinumresistant ovarian cancer. Patients who relapse more than 6 months are considered as platinum-sensitive 
ovarian cancer. Furthermore, patients were divided into first-line maintenance treatment group, secondline maintenance treatment group and monotherapy after multi-line( $\geq 3$ lines) treatment group in accordance with indications approved by Food and Drug Administration (FDA). The rest of patients that were not applied in the scope of indications were classified as exploratory therapy group.

\section{Drug administration}

Initially, all patients were orally given the standard dose of $300 \mathrm{mg}$ Olaparib twice a day, and most patients were discontinued upon the progressive disease (PD) or intolerable adverse reaction. A few patients were treated with anti-angiogenic drugs as the disease progressed. Adverse events were graded according to National Cancer Institute Common Terminology Criteria for Adverse Events (NCI CTCAE) version 4.0. There were 8 patients who reduced the dose of Olaparib after the evaluation of adverse events, and 4 patients with severe hematological adverse events continued Olaparib treatment after relief.

\section{Statistical analysis}

Descriptive statistics of clinical and demographic characteristics were summarized. Safety analysis was performed among all enrolled patients who received greater than or equal one dose. AEs were graded according to NCI CTCAE version 4.0. The baseline of CA125 was used as the reference value, and all data were converted to natural logarithm. Short-term efficacy was classified as CR, partial remission (PR), $\mathrm{SD}$ and $\mathrm{PD}$ by modified Response Evaluation Criteria in Solid Tumors version 1.1(RECIST 1.1).

\section{Results}

\section{Patients' characteristics}

The average age of the 28 included patients was 59 years (range 36-79), and 92.9\% were ovarian cancer, $7.1 \%$ were diagnosed as fallopian tube cancer. The ECOG scores of all patients were 0-1. Advanced ovarian cancer, known as FIGO III or IV, affected 19 $(67.9 \%)$ and $3(10.7 \%)$ of patients, respectively. 25 patients suffered from high-grade serous adenocarcinoma, and the remaining 3 cases were endometrioid carcinoma, mixed serous and endometrioid carcinoma and mixed serous and mucious carcinoma. 39.3\% patients harbored BRCA $1 / 2$ mutation, also $39.3 \%$ patients had BRCA wild type. Baseline characteristics of eligible patients were summarized in Table 1. The family history of the patients was classified according to their BRCA status (Table 2).

\section{Patients' classification}

In this study, there were 11 patients diagnosed with platinum sensitive ovarian cancer, 13 patients with platinum resistance ovarian cancer, and 4 patients with unknown platinum reaction. Subsequently, we divided the enrolled patients into 4 groups. Among them, 2 patients received first-line maintenance treatment, 10 received second-line maintenance treatment, 6 received single-drug treatment after third-line chemotherapy, and 10 received exploratory drugs, 6 of whom were patients with BRCAwt and BRCA unknown after multi-line( $\geq 3$ lines $)$ treatment. The other three patients harbored BRCAm were the first recurrence platinum sensitivity ovarian cancer or fallopian tube cancer, one of them recurred after maximal cytoreductive surgery and platinum-based chemotherapy, the two patients recurred after only maximal cytoreductive surgery without postoperative chemotherapy. The last patient was treated with Olaparib after only chemotherapy without surgery. In monotherapy group, four platinumresistant patients harbored gBRCA mutations after multiline chemotherapy used Olaparib. One of the other two platinum-resistant patients in monotherapy group carried the suspected pathogenic frameshift mutation c.7198 Inst (p.Gly2400Valfs*12p), and the other one carried the unknown missense mutation c.112 $\mathrm{g}>\mathrm{A}$ (p.Glu38Lys), both of which were possible pathological mutations. Antiangiogenic agents were added to 4 patients with the progression of the disease.

\section{Drug related AEs}

The most common all grade adverse events were fatigue or asthenia (60.70\%), decreased appetite (42.9\%), anemia (42.9\%), nausea (39.3\%), arthralgia (32.1\%) and vomiting (25\%). Most patients had grade 1 or 2 AEs. Adverse reactions of grade 3 or 4 were observed in few cases. Anemia, abdominal pain and thrombocytopenia occurred in three patients, two patients and one patient, respectively. Other AEs included diarrhea (10.7\%), constipation (17.9\%), dysgeusia (17.9\%), dizziness (10.7\%), cough (10.7\%), back pain (14.3\%) and dyspnea (3.6\%). These AEs occurred within 3 months after administration.

We also identified several AEs that have never been reported previously, including abdominal distention (14.3\%), decreased blood pressure (3.6\%), increased body hair (3.6\%), thirsty (3.6\%), burning sensation of stomach (3.6\%) and leg swelling (3.6\%). The occurrence time of the newly AEs was similar to that of the known AEs. Similarly, most patients had mild AEs. Only 1 patient with declined blood pressure stopped Olaparib treatment. 
Table 1 Baseline characteristics in 28 patients. Values are reported as frequency ( $\mathrm{n}[\%])$ or as mean (range)

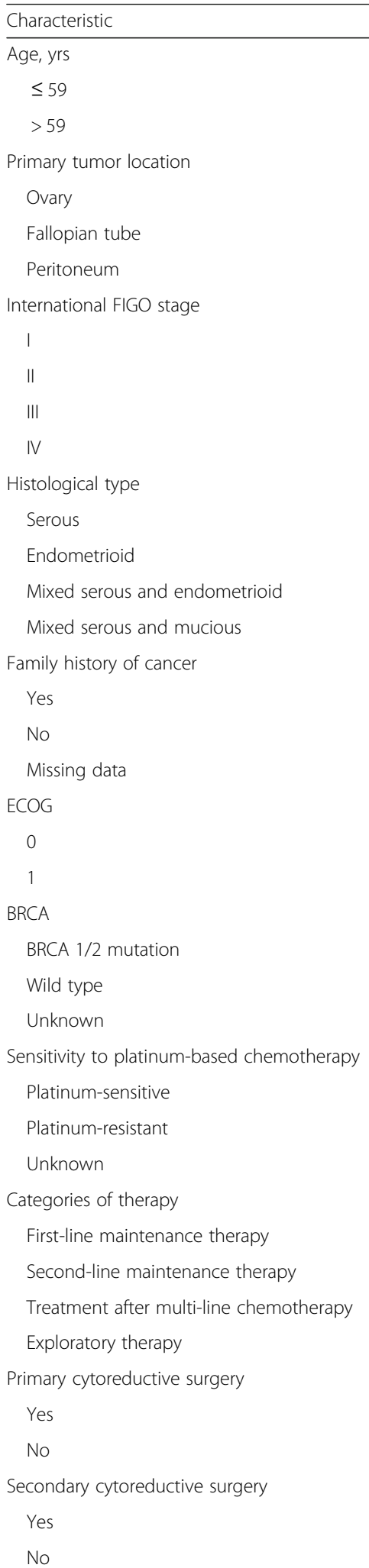

$13(46.4)$

$15(53.6)$

$26(92.9)$

$2(7.1)$

$0(0)$

$4(14.3)$

$2(7.1)$

19 (67.9)

$3(10.7)$

$25(89.3)$

1 (3.6)

1 (3.6)

1 (3.6)

$10(35.7)$

$15(53.6)$

$3(10.7)$

$18(64.3)$

$10(35.7)$

11 (39.3)

$11(39.3)$

$6(21.4)$

11 (39.3)

$13(46.4)$

$4(14.3)$

$2(7.1)$

$10(35.7)$

$6(21.4)$

$10(35.7)$

27 (96.4)

1 (3.6)

$6(21.4)$

$22(78.6)$

Number of patients (percent)

Table 1 Baseline characteristics in 28 patients. Values are reported as frequency (n [\%]) or as mean (range) (Continued)

\begin{tabular}{lc}
\hline Characteristic & Number of patients (percent) \\
\hline Combination of anti-angiogenic agents & \\
Yes & $4(14.3)$ \\
No & $24(85.7)$ \\
\hline
\end{tabular}

Abbreviations: FIGO, International Federation of Gynecology and Obstetrics; ECOG, Eastern Cooperative Oncology Group

AEs were usually managed by dose interruption or dose reduction, rather than discontinuation. 3 patients discontinued treatment, 8 patients received reduced treatment, and 4 patients interrupted treatment after the alleviation of AEs (Table 3). Short-term efficacy was not affected among 2 of 8 patients who took low dose of Olaparib. Further, the short-term clinical outcome was not affected for 3 of the 4 patients who orally received $150 \mathrm{mg}$ of Olaparib twice a day. After they were recovered from the AEs, these three patients all received reduced dose of Olaparib. Over the following 3 months, the levels of CA125 were elevated but the imaging showed no recurrence among 2 out 3 patients.

The most common AEs that led to interruption were anemia and decreased blood pressure. 2 of 3 patients who discontinued treatment had severe anemia and 1 also had severe leucopenia. The major reasons for patients who reduced the amount of Olaparib were digestive tract reactions, bone marrow suppression including anemia, leucopenia and thrombocytopenia and abdominal pain.

\section{Short-term efficacy}

In exploratory therapy group, 2 patients achieved CR, 3 patients achieved SD, whereas 5 patients had disease progressed. One patient took Olaparib when she firstly recurred after merely maximal cytoreductive surgery without postoperative chemotherapy and another patient was treated with Olaparib after only chemotherapy without surgery. These two patients with CR were followed up for 3 months and

Table 2 Classification of family history according to BRCA status

\begin{tabular}{ll}
\hline BRCA status & Family history of cancer \\
\hline BRCA unknown & Skin cancer, leukemia \\
BRCA1m & Ovarian cancer, pancreatic \\
& cancer, gastric cancer, \\
& cervical cancer, esophageal \\
& cancer
\end{tabular}

BRCA2m

Ovarian cancer, lung cancer, rectal cancer, retroperitoneal neoplasms

Gastric cancer, lymphoma, gingival cancer, breast cancer, rectal cancer 
Table 3 Summary of Adverse Events

\begin{tabular}{|c|c|c|}
\hline \multirow[t]{2}{*}{ Adverse Event } & Any Grade & Grade 3 or 4 \\
\hline & \multicolumn{2}{|c|}{ number of patients (percent) } \\
\hline Fatigue or asthenia & $17(60.7)$ & $0(0)$ \\
\hline Anemia & $12(42.9)$ & $3(10.7)$ \\
\hline Decreased appetite & $12(42.9)$ & $0(0)$ \\
\hline Nausea & $11(39.3)$ & $0(0)$ \\
\hline Arthralgia & $9(32.1)$ & $0(0)$ \\
\hline Vomiting & $7(25.0)$ & $0(0)$ \\
\hline Abdominal pain & $5(17.9)$ & $2(7.1)$ \\
\hline Constipation & $5(17.9)$ & $0(0)$ \\
\hline Dysgeusia & $5(17.9)$ & $0(0)$ \\
\hline Neutropenia & $5(17.9)$ & $0(0)$ \\
\hline Thrombocytopenia & $4(14.3)$ & $1(3.6)$ \\
\hline Back pain & $4(14.3)$ & $0(0)$ \\
\hline Diarrhea & $3(10.7)$ & $0(0)$ \\
\hline Dizziness & $3(10.7)$ & $0(0)$ \\
\hline Upper abdominal pain & $3(10.7)$ & $0(0)$ \\
\hline Cough & $3(10.7)$ & $0(0)$ \\
\hline Dyspnea & 1 (3.6) & $0(0)$ \\
\hline Headache & $0(0)$ & $0(0)$ \\
\hline Dyspepsia & $0(0)$ & $0(0)$ \\
\hline \multicolumn{3}{|l|}{ Newly observed } \\
\hline Abdominal distention & $4(14.3)$ & $0(0)$ \\
\hline Decreased blood pressure & 1 (3.6) & $0(0)$ \\
\hline Skin rash & $1(3.6)$ & $0(0)$ \\
\hline Increased body hair & $1(3.6)$ & $0(0)$ \\
\hline Thirsty & $1(3.6)$ & $0(0)$ \\
\hline Burning sensation of stomach & $1(3.6)$ & $0(0)$ \\
\hline Leg swelling & $1(3.6)$ & $0(0)$ \\
\hline Led to discontinuation of intervention & $3(10.7)$ & - \\
\hline Led to dose reduction & $8(28.6)$ & - \\
\hline Led to dose interruption & $4(14.3)$ & - \\
\hline
\end{tabular}

Adverse events were graded according to National Cancer Institute Common Terminology Criteria for Adverse Events(NCl CTCAE), version 4.0
Table 4 Short-term efficacy of exploratory therapy

\begin{tabular}{ll}
\hline Short-term efficacy & Number of patients (Percent) \\
\hline Complete response (CR) & $2(20.0)$ \\
Partial response (PR) & $0(0)$ \\
Stable disease (SD) & $3(30.0)$ \\
Progressive disease (PD) & $5(50.0)$ \\
\hline
\end{tabular}

Short-term efficacy was classified by modified Response Evaluation Criteria in Solid Tumors version 1.1(RECIST 1.1)
Table 5 Short-term efficacy of monotherapy after multi-line chemotherapy

\begin{tabular}{ll}
\hline Short-term efficacy & Number of patients (Percent) \\
\hline Complete response (CR) & $0(0)$ \\
Partial response (PR) & $0(0)$ \\
Stable disease (SD) & $1(33.3)$ \\
Progressive disease (PD) & $2(66.7)$ \\
\hline
\end{tabular}

4 months, respectively (Table 4 ). Three patients with SD were followed up for 6 months, 7 months and 2 months, respectively. The first patient used Olaparib also when she recurred after maximal cytoreductive surgery and platinum-based chemotherapy, the second patient was treated with Olaparib when she relapsed after only maximal cytoreductive surgery without postoperative chemotherapy and the third platinum-resistant recurrent ovarian cancer patient with BRCAw took Olaparib after multi-line therapy.

In the multi-line therapy group, one patient was assessed as SD after monotherapy for 3 months (Table 5). Three platinum-resistant recurrent ovarian cancer patients added anti-angiogenic agents (Cediranib was purchased by herself from abroad or Apatinib was produced in Jiangsu HengRui Pharmaceutical co. LTD) after progressed, 2 patients fortunately achieved SD in seven months and three months, respectively (Table 6).

Another patient found a continuous increase in CA125 level during the second-line maintenance treatment, and a persistence decrease in CA125 level occurred when Apatinib was added under the strong personal will of herself.

The imaging evaluation of multi-line therapy group and exploratory therapy group on the basis of RECIST 1.1 were shown in Fig. 1. Meanwhile, CA125 level were measured among various groups with different BRCA mutation status in Figs. 2, 3, 4, 5 and 6. The levels of CA125 in the first-line maintenance and the second-line maintenance groups were comparatively lower than that in the other two groups.

\section{Disscusion}

Molecular targeted therapy is currently the central issue of cancer treatment. Targeted therapy for non-small cell lung cancer has become one of well-accepted treatment

Table 6 Short-term efficacy of monotherapy combined with anti-angiogenic agents after PD with monotherapy

\begin{tabular}{ll}
\hline Short-term efficacy & Number of patients (Percent) \\
\hline Complete response (CR) & $0(0)$ \\
Partial response (PR) & $0(0)$ \\
Stable disease (SD) & $2(66.7)$ \\
Progressive disease (PD) & $1(33.3)$ \\
\hline
\end{tabular}




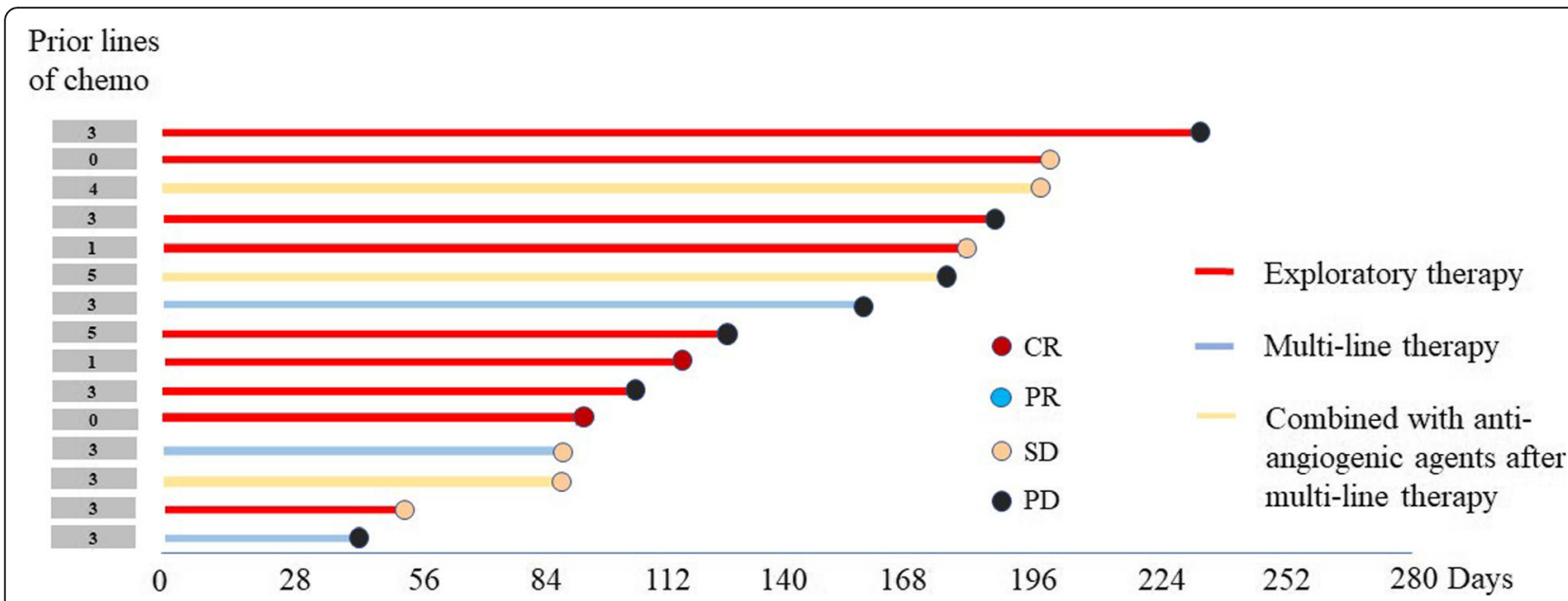

Fig. 1 Water plot of best response by RECIST1.1. Note: This is a water plot of the latest imaging evaluation in multi-line therapy group and exploratory therapy group, including 6 patients in multi-line therapy group and 10 patients in exploratory therapy group. Three patients in multiline treatment group added anti-angiogenic drugs when developed progressive disease, two of them achieved stable disease after combined two drugs. In exploratory therapy group one platinum-resistant ovarian cancer patient with non-BRCA mutation achieved progressive disease after using Olaparib for one month and she died soon, which was not shown in the figure. Finally, the short-term efficacy of 9 patients in exploratory therapy group were shown in the figure

principles in the worldwide [12]. Ovarian cancer is the most fatal gynecologic malignant tumor. PARP inhibitors have revolutionized the traditional treatment strategy of ovarian cancer. The results of two phase III trials, SOLO1 and SOLO2, showed that the Olaparib as the first-line/second-line maintenance therapy could significantly improve the progression-free survival and reduce the risk of disease recurrence and mortality $[9,10]$. Also, in previous study, it was also found that Olaparib monotherapy after the multi-line treatment in patients with germline BRCA mutations could increase the tumor response rate [7]. SOLO3 was reported in 2019 ASCO, suggesting that that multi-line platinum-sensitive patients with germline mutations could be significantly benefit from Olaparib (ORR72\%, PFS $13.2 \mathrm{~m}$ vs. $8.5 \mathrm{~m}$ ) [13]. The study of CLIO indicated that the efficiency of multi-line platinum-resistant patients was $18 \%$, which is superior to $6 \%$ of standard chemotherapy [14]. These latest findings further validated that PARP inhibitors could offer a non-chemotherapeutic treatment option for platinum-sensitive or platinum-resistant relapse patients. EVOLVE study also showed that the addition of cediranib treatment reached the expected efficacy for patients with PARPi resistance [15].

Among the four groups in this study, all patients had AEs, and the most common adverse reaction were fatigue, loss of appetite, anemia, nausea and vomiting, and the majority patients had grade 1-2 AEs. Seventeen percentage of patients developed grade 3-4 AEs, including severe anemia, abdominal pain, and thrombocytopenia. AEs were usually managed by dose interruption or dose reduction, rather than discontinuation. In our study, the median time

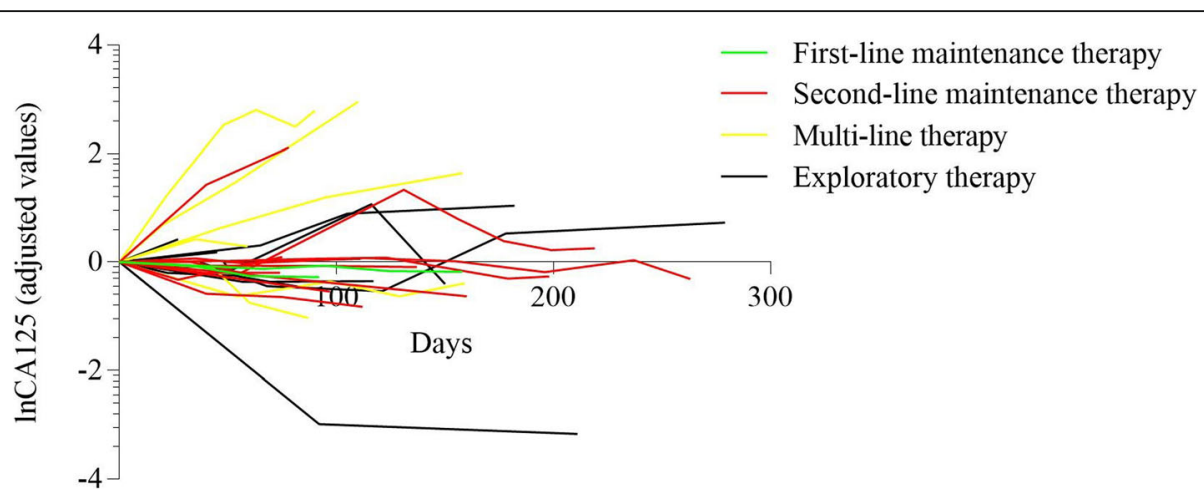

Fig. 2 CA125 values in each group. Note: The CA125 level of the first follow-up was used as the reference value, and all data were converted to natural logarithm. CA125 follow-up data were obtained from 2 patient in the first-line maintenance treatment group, 10 patients in the secondline maintenance treatment group, 6 patients in the multi-line therapy group, and 10 patients in the exploratory therapy group 


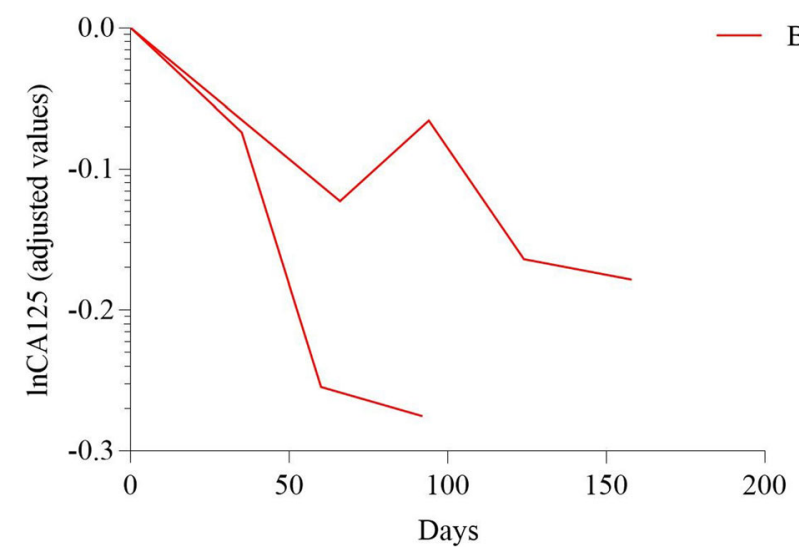

Fig. 3 CA125 in patients with different BRCA status in the first-line maintenance group. Note: Two patients with BRCA mutation in the first-line maintenance treatment group was followed up with CA125

of occurrence of AEs and SAEs was consistent with previous studies and SOLO1 from China cohort [16]. However, the incidence of serious adverse reactions was lower, which may be due to the relatively small number of subjects in our study. For the first time, we also identified a few AEs during the follow-up which have not been reported. A platinum-sensitive recurrence patient had a substantial reduction of blood pressure after 4 months of Olaparib monotherapy. The patient's blood pressure went back up when she was given in half of the standardized dosage. The other mild AEs first identified in our study also included abdominal distention, increased body hair, thirsty, burning sensation of stomach and leg swelling. Interestingly, all patients with these unreported AEs were found in patients harbored BRCA mutations, and whether such BRCA mutations played a role in AEs the occurrence of AEs remains to be explored.

A multicentre, single-arm, phase 2 study that evaluated the safety and activity of Niraparib in patients with relapsed ovarian cancer who were treated with three or more previous chemotherapy regimens. Patients were orally received $300 \mathrm{mg}$ of Niraparib once per day. Since the patients showed reduced platelet, the dose was adjusted from $300 \mathrm{mg}$ to $200 \mathrm{mg}$. AEs were significantly reduced, and the efficacy of Niraparib was not affected by AEs [17]. Similarly, we also found that some patients who received oral reduction dose of Olaparib did not affect the short-term effect in our cases. It is suggested that Chinese people or population with a baseline BMI or baseline AUC who received lower dose of Olaparib could be benefit from it and reduced the incidence of treatmentemergent adverse events.

We also analyzed the short-term efficacy of the posterior monotherapy group and exploratory treatment group, and found that 1 patient in the posterior monotherapy group achieved stability. 2 patients with BRCA mutant in this group progressed who achieved stable disease after adding anti-angiogenic drugs. 1 patient was platinum-sensitive recurrent ovarian

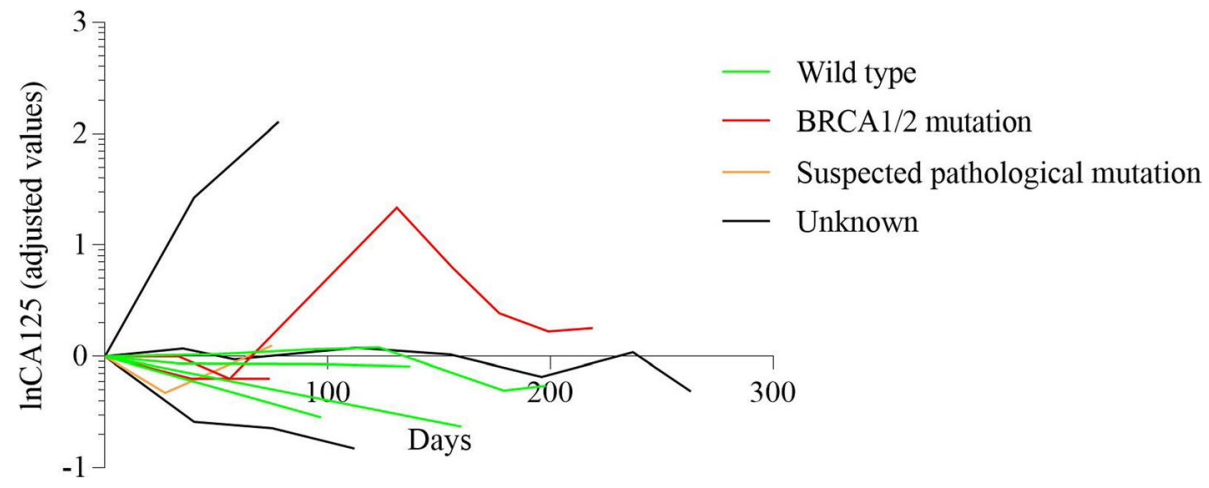

Fig. 4 CA125 in patients with different BRCA status in the second-line maintenance group. Note: There were two patients with BRCA mutation, four patients with BRCA wild-type, one patient with BRCA suspected pathological mutation and also three patients with BRCA status unknown in this group 


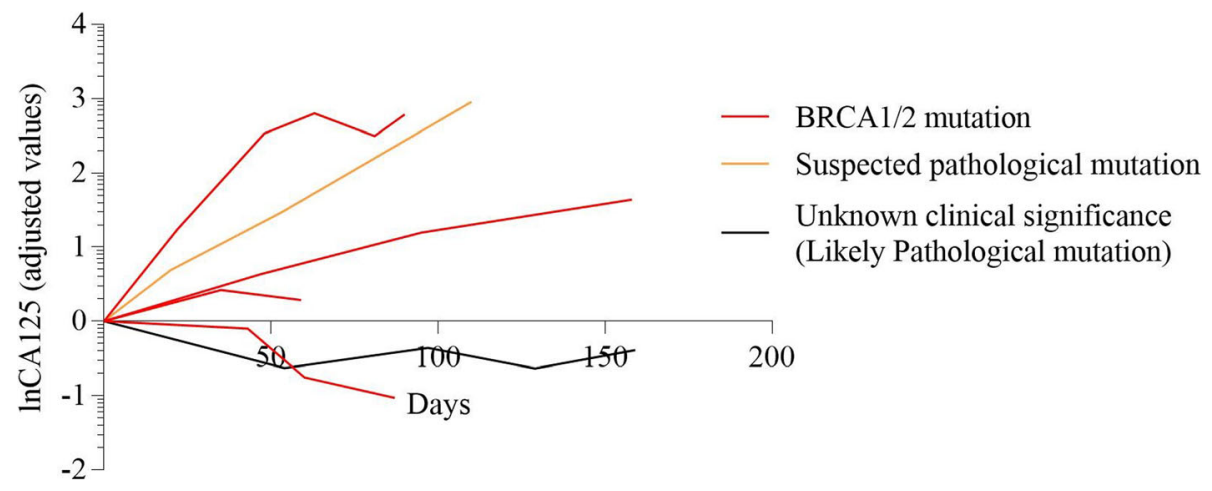

Fig. 5 CA125 in patients with different BRCA status in multi-line therapy group. Note: There were four patients with BRCA mutant, one patient with BRCA suspected pathological mutation and also one patient with BRCA unknown clinical significance mutation in this group

cancer and the other was platinum-resistant recurrent ovarian cancer, which were consistent with the results of EVOLVE [15]. Interestingly, in the exploratory treatment group, we found that one patient achieved CR after using Olaparib when she firstly recurred after merely maximal cytoreductive surgery without postoperative chemotherapy and another patient also achieved CR after using Olaparib when she only took chemotherapy without suffering surgery. The benefits of these exploratory drugs provided us with new evidence for clinical trials in the future.

BRCA mutations that are closely related to the potency of PARP inhibitors under some circumstances. Previous studies have shown that the risk of ovarian cancer in the general population is $1-2 \%$, while the risk of population with BRCA1 mutant and BRCA2 mutant are $39-63 \%$ and $16.5-27 \%$, respectively [18].BRCA mutant are closely associated with breast cancer and may be also related to prostate cancer [19], pancreatic cancer [20], and cutaneous melanoma [21]. In this study, family history of cancer for patients was also considered. It was found that the family with BRCA1 mutant had pancreatic cancer, gastric cancer, cervical cancer and esophageal cancer, while the family with BRCA2 mutant had lung cancer, rectal cancer and close relatives of retroperitoneal tumor. The results suggest that BRCA mutant may be associated with tumorigenesis in a variety of cancers. And further research is needed to confirm the heredity in population with BRCA mutant. Based on the clinical benefits of PARP inhibitors in ovarian and pancreatic cancer with BRCA mutant [22], whether the indications for PARP inhibitors for tumors associated with BRCA mutations should be further explored.

\section{Conclusion}

In conclusion, our study evaluated the side effects and short-term efficacy in ovarian cancer patients who were treated with Olaparib, in the real-world setting. This study mainly focused on AEs and SAEs of patients that are consistent with the previously

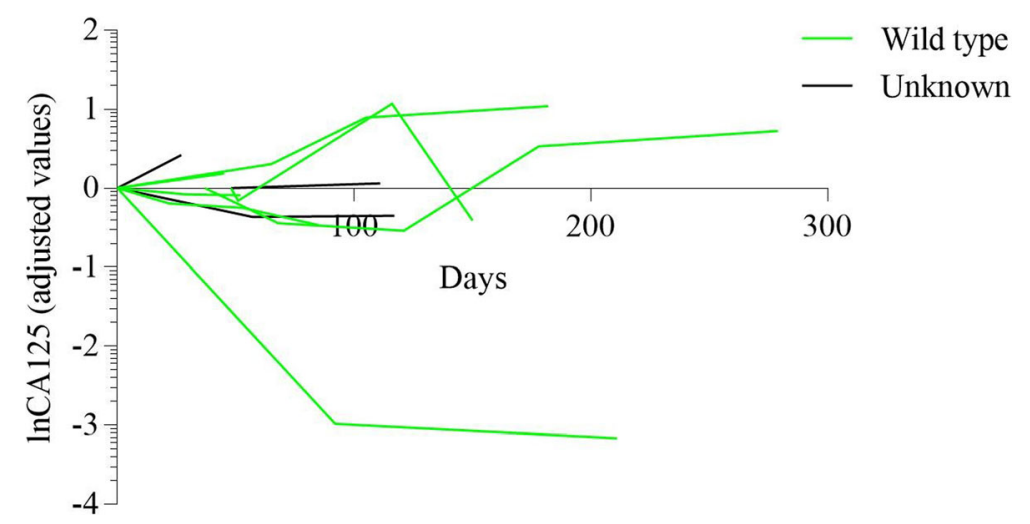

Fig. 6 CA125 in patients with different BRCA status in exploratory therapy group. Note: There were seven patients with BRCA wild-type and three patients with BRCA status unknown in this group 
published results. Nevertheless, we also observed several unreported AEs. In addition, we are concerned about the clinical efficacy of Olaparib, although there is a certain clinical remission rate within short time, but the clinical outcome for these patients with ovarian cancer needs to be further followed up. Also, the benefits in exploratory treatment group provided new potential indication to PARPi-related clinical trials.

\section{Abbreviations}

AE: Adverse Events; EOC: Epithelial Ovarian Cancer; PARP: Poly Adenosine diphosphate Ribosome Polymerase; RECIST: Response Evaluation Criteria in Solid Tumors; SAE: Serious Adverse Events

\section{Acknowledgments}

We sincerely appreciate Jia Chen, Fei Deng, Zhiqin Dai, Hong Pei, Xiaofeng Sun, Jinhuang Wang, Yuzhong Wu, Xuehui Zhou and Yibing Zhao(This list is in no particular order of importance and sequenced by first letter of last name).

\section{Authors' contributions}

Jing Ni participated in the design of present study and drafted the manuscript. Xianzhong Cheng and Xia Xu carried out the cases recruit of present study. Jing Ni participated in the cases recruit of present study. Rui Zhou carried out statistical analysis. Wenwen Guo participated in the statistical analysis and drafted the manuscript. Xiaoxiang Chen designed of the study, performed the statistical analysis and revised the manuscript. All authors read and approved the final manuscript.

\section{Funding}

This study was supported by grants from the National Natural Science Foundation of China (No. 81472441, 81501205).

\section{Availability of data and materials}

We would not share the data and material used in this manuscript, because we need them for further research.

\section{Ethics approval and consent to participate}

This retrospective study was approved by the institutional review board of Jiangsu Cancer Hospital, Nanjing Medical University, China. The informed consent requirement was waived. The committee's reference number was Jiangsu Cancer Hospital's Ethical Committee 2016-05-01.

\section{Consent for publication}

Not applicable.

\section{Competing interests}

The authors declare that they have no competing interests.

\author{
Author details \\ 'Department of Gynecologic Oncology, The Affiliated Cancer Hospital of \\ Nanjing Medical University, Jiangsu Cancer Hospital, Jiangsu Institute of \\ Cancer Research, 42\# Baiziting Street, Nanjing, Jiangsu 210009, People's \\ Republic of China. ${ }^{2}$ Department of Chemotherapy, Nanjing Medical \\ University Affiliated Cancer Hospital, Jiangsu Cancer Hospital, Jiangsu \\ Institute of Cancer Research, 42\# Baiziting Street, Nanjing, Jiangsu 210009 \\ People's Republic of China. ${ }^{3}$ Department of Pathology, The Second Affiliated \\ Hospital of Nanjing Medical University, Nanjing, Jiangsu 210009, People's \\ Republic of China.
}

Received: 16 August 2019 Accepted: 19 November 2019 Published online: 28 November 2019

\section{References}

1. Gupta KK, Gupta VK, Naumann RW. Ovarian cancer: screening and future directions. Int J Gynecol Cancer. 2019;29(1):195-200.

2. Siegel RL, Miller KD, Jemal A. Cancer statistics, 2019. CA Cancer J Clin. 2019; 69(1):7-34.
3. Chen W, et al. Cancer statistics in China, 2015. CA Cancer J Clin. 2016;66(2): $115-32$.

4. Broekman KE, et al. Clinical benefit of controversial first line systemic therapies for advanced stage ovarian cancer - ESMO-MCBS scores. Cancer Treat Rev. 2018;69:233-42.

5. Kim A, et al. Therapeutic strategies in epithelial ovarian cancer. J Exp Clin Cancer Res. 2012;31:14

6. Schiewer MJ, et al. PARP-1 regulates DNA repair factor availability. EMBO Mol Med. 2018:10(12)

7. Kaufman B, et al. Olaparib monotherapy in patients with advanced cancer and a germline BRCA1/2 mutation. J Clin Oncol. 2015;33(3):244-50.

8. Ledermann $\mathrm{JA}$, et al. Overall survival in patients with platinum-sensitive recurrent serous ovarian cancer receiving olaparib maintenance monotherapy: an updated analysis from a randomised, placebo-controlled, double-blind, phase 2 trial. Lancet Oncol. 2016;17(11):1579-89.

9. Pujade-Lauraine $\mathrm{E}$, et al. Olaparib tablets as maintenance therapy in patients with platinum-sensitive, relapsed ovarian cancer and a BRCA1/2 mutation (SOLO2/ENGOT-OV21): a double-blind, randomised, placebo-controlled, phase 3 trial. Lancet Oncol. 2017;18(9):1274-84.

10. Moore K, et al. Maintenance Olaparib in patients with newly diagnosed advanced ovarian Cancer. N Engl J Med. 2018;379(26):2495-505.

11. Washington C, Gunderson CC, Moore KN. Update in the use and evaluation of poly (ADP-ribose) polymerase inhibitors in epithelial ovarian cancer: current and pending clinical research. Curr Opin Obstet Gynecol. 2019:31(1): 4-11.

12. Camidge DR, Doebele RC, Kerr KM. Comparing and contrasting predictive biomarkers for immunotherapy and targeted therapy of NSCLC. Nat Rev Clin Oncol. 2019;16(6):341-55.

13. Penson RT, et al. Olaparib monotherapy versus (vs) chemotherapy for germline BRCA-mutated (gBRCAm) platinum-sensitive relapsed ovarian cancer (PSR OC) patients (pts): Phase III SOLO3 trial. 2019. Proc Am Soc Clin Oncol.

14. Vanderstichele A, et al. Randomized phase /l CLIO study on olaparib monotherapy versus chemotherapy in platinum-resistant ovarian cancer. 2019. Proc Am Soc Clin Oncol.

15. Lheureux S, et al. Evolve: A post PARP inhibitor clinical translational phase II trial of cediranib-olaparib in ovarian cancer-A Princess Margaret ConsortiumGCIG Phase II Trial. 2019. Proc Am Soc Clin Oncol.

16. Wu L, et al. Olaparib maintenance therapy in patients (pts) with a BRCA1 and/ or BRCA2 mutation (BRCAm) and newly diagnosed advanced ovarian cancer (OC): SOLO1 China cohort. 2019. Proc Am Soc Clin Oncol.

17. Moore KN, et al. Niraparib monotherapy for late-line treatment of ovarian cancer (QUADRA): a multicentre, open-label, single-arm, phase 2 trial. Lancet Oncol. 2019;20(5):636-48,

18. Hawsawi YM, et al. Mol Genet Genomic Med. 2019:e879.

19. Lecarpentier J, et al. Prediction of breast and prostate Cancer risks in male BRCA1 and BRCA2 mutation carriers using polygenic risk scores. J Clin Oncol. 2017;35(20):2240-50.

20. Cicenas J, et al. KRAS, TP53, CDKN2A, SMAD4, BRCA1, and BRCA2 Mutations in Pancreatic Cancer. Cancers (Basel). 2017:9(5).

21. Gumaste PV, et al. Skin cancer risk in BRCA1/2 mutation carriers. $\mathrm{Br}$ Dermatol. 2015;172(6):1498-506.

22. Kindler $\mathrm{HL}$, et al. Olaparib as maintenance treatment following first-line platinum-based chemotherapy (PBC) in patients (pts) with a germline BRCA mutation and metastatic pancreatic cancer (mPC): Phase III POLO trial. Proc Am Soc Clin Oncol. 2019.

\section{Publisher's Note}

Springer Nature remains neutral with regard to jurisdictional claims in published maps and institutional affiliations. 